\title{
Extracapsular lens extraction and posterior chamber intraocular lens insertion combined with trabeculectomy
}

\author{
JEFFREY L JAY \\ From the Tennent Institute of Ophthalmology, University of Glasgow
}

SUMMARY Trabeculectomy for primary glaucoma was successfully combined with extracapsular lens extraction and insertion of a Pearce tripod posterior chamber lens in seven eyes of five patients. The results were similar to those expected from each component of the operation when performed alone, and all eyes achieved visual acuity of $6 / 9$ or better with control of intraocular pressure below $22 \mathrm{mmHg}$ without supplementary medical treatment. The incidence of mild anterior uveitis (three out of seven eyes) and hyphaema (two out of seven eyes) was greater than usual with this type of cataract extraction but no more than expected after trabeculectomy. Mydriatic drops may be used to treat postoperative iritis without danger to the stability of this pattern of implant. In addition the anterior chamber cannot become shallow, as the rigid legs of this lens extend behind the iris beyond the periphery of the cornea and prevent forward movement of the implant even if there is excessively free drainage of aqueous after the operation.

In recent years authors from many countries have considered it an advantage to combine simultaneous trabeculectomy with lens extraction when cataract surgery becomes necessary in patients with glaucoma. ${ }^{1-9}$ Earlier reports justified this combined operation only in cases where the intraocular pressure had been difficult to control medically, but recently the indications have widened to include cases where medical control has appeared satisfactory before cataract surgery. The risks and successes of the combined procedure are those of each component separately, and it reduces the chance of having to deal with the considerable problems in management of uncontrolled glaucoma in an aphakic eye.

The safety and optical advantages of intraocular lens implantation are now widely recognised, and, although Stiegler" has reported favourable results combining the insertion of a Kelman II anterior chamber lens when intracapsular extraction is combined with trabeculectomy, many surgeons would still hesitate to add lens implantation to the operation of cataract extraction combined with trabeculec-

Correspondence to Dr J L Jay, Tennent Institute of Ophthalmology, Church Strect, Glasgow G11 6NT. tomy. In 1979 Percival" succinctly summarised the practical problems, and de Heer ${ }^{12}$ reviewed the limited reports on various lens types.

This paper describes the initial experience of satisfactory insertion of a single plane, rigid posterior chamber intraocular lens when trabeculectomy is combined with extracapsular lens extraction.

\section{Material and methods}

SURGICAL TECHNIQUE

The operation is essentially a combination of Watson's trabeculectomy ${ }^{13}$ and extracapsular lens extraction with insertion of a sutureless RaynerPearce vaulted tripod posterior chamber lens in the capsular bag. ${ }^{14}$ An adequate operating microscope with coaxial illumination is therefore essential to display the dissection of the lens capsule against the red reflex. Chloramphenicol eye drops are applied four times in the 24 hours before operation, and the pupil is fully dilated with $1 \%$ cyclopentolate and $10 \%$ phenylephrine immediately before surgery. This is supplemented with a subconjunctival injection of $0.25 \mathrm{ml}$ of Mydricaine No. 2 (atropine, procaine, adrenaline), once the patient has been anaesthetised, so that full mydriasis is maintained during the 
intraocular dissection. It is wise to avoid dilating the pupil as part of the admission examination, because this preliminary exposure often renders the pupil refractory to subsequent dilatation at the time of operation. An outer sceral flap $4 \times 4 \mathrm{~mm}$ is fashioned under a fornix based conjunctival flap, and the remainder of the corneoscleral incision for cataract extraction extends a further $6 \mathrm{~mm}$ to one side of the trabeculectomy site, so that the peripheral iridectomy at the trabeculectomy fistula will not coincide with the tip of the upper leg of the implant. After raising the superficial scleral flap and completing the corneoscleral ab-externo groove, the anterior chamber is entered in the depth of the groove with an irrigating cystitome through a puncture incision small enough to ensure a watertight seal. The D shaped anterior capsulotomy is then completed to fashion a deep inferior pocket before the inner corneoscleral block of the trabeculectomy is excised. Peripheral iridectomy is performed at the trabeculectomy site, and the trabeculectomy opening is then extended along the ab-externo groove incorporating the entry puncture used for capsulotomy. Thereafter the anterior capsule is removed, the pupil is swept behind the upper denuded part of the lens, and the lens is removed with an irrigating vectis. Any remaining cortex is aspirated manually with a coaxial aspirationirrigation cannula, care being taken to preserve the posterior capsule. A sutureless Pearce tripod vaulted intraocular lens of suitable power is inserted into the inferior capsular pocket, and the upper leg $(6.5 \mathrm{~mm})$ is slipped behind the pupil into the iridociliary sulcus at the 12 o'clock meridian. The incision is closed with 10-0 nylon interrupted sutures at the corners of the trabeculectomy flap and along the corneoscleral wound.

Postoperative treatment consists of chloramphenicol and betamethasone drops applied four times daily with a mydriatic regimen of once daily intensive dilatation of the pupil with cyclopentolate and phenylephrine drops combined with a regular prescription for cyclopentolate or atropine as necessary. The eye is bandaged for the first two days, and the patient is discharged between the 3rd and 6th day, depending on home circumstances.

\section{PATIENTS}

The operation was performed on seven eyes of five consecutive patients who had primary glaucoma requiring treatment and who were otherwise suitable for extracapsular lens extraction with intraocular lens implantation. There were four female and one male patient, whose ages ranged between 68 and 81 years (mean 73 years). Four had primary open angle glaucoma and one had chronic angle closure glaucoma with $30 \%$ peripheral anterior synechiae.
Visual field analysis with a Tubingen perimeter had suggested arcuate visual field defects diagnostic of glaucoma in four patients when first examined and before the development of dense cataract. The fifth patient had advanced cataract when first examined and accurate visual field testing was not possible. For each eye the maximum intraocular pressure without medical therapy ranged from 24 to $36 \mathrm{mmHg}$ (average $28 \mathrm{mmHg}$ ). No eye had had previous surgery.

\section{Results}

Patients have been followed up for two to 36 months (mean 18 months). In all eyes vision was restored to $6 / 9$ or better, and the intraocular pressure was reduced to below $22 \mathrm{mmHg}$ without supplementary medical therapy. Fig. 1 shows the maximum untreated preoperative intraocular pressure and the postoperative levels. The effect of this combined operation, as demonstrated by the straight line relating the reduction in intraocular pressure to the initial maximum untreated pressure, is similar to that found when trabeculectomy alone was carried out in

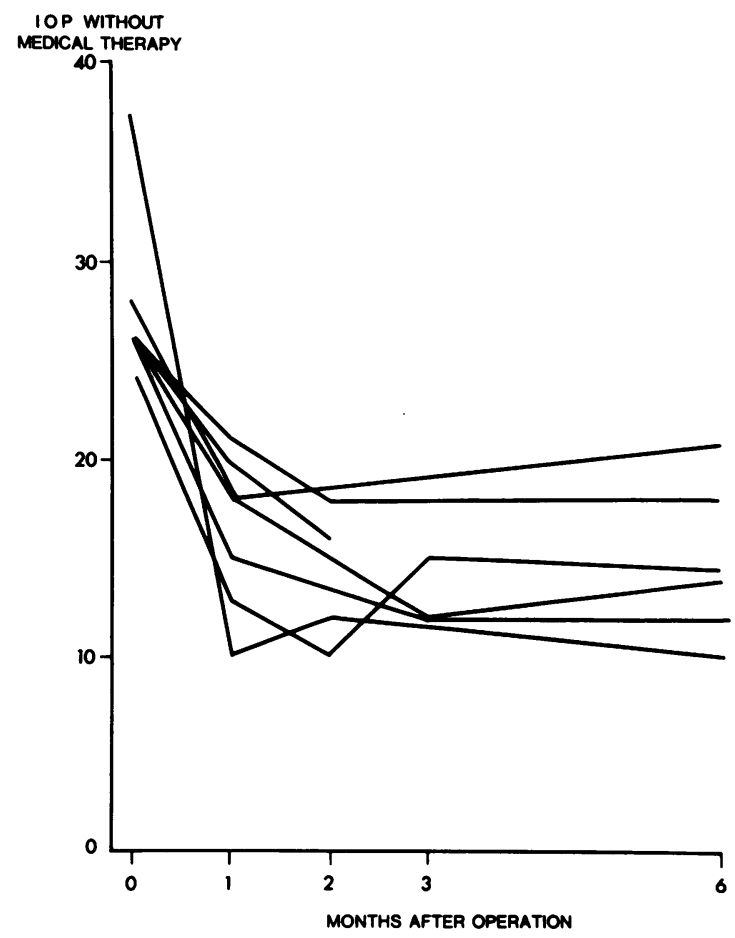

Fig. 1 Reduction of intraocular pressure from untreated preoperative levels after trabeculectomy combined with extracapsular lens extraction and insertion of a Pearce tripod posterior chamber lens. No medication was required to reduce the postoperative intraocular pressure. 
phakic eyes with primary open angle glaucoma (Fig. 2).

The postoperative course was as expected with hyphaema (two cases) and iritis (three cases) occurring with the frequency and severity observed after trabeculectomy alone. The hyphaemas resolved within two days in both cases, and in all cases the anterior chamber inflammation settled quickly so that the postoperative eye drops could be discontinued within six weeks. It was, however, impossible to prevent minor posterior synechiae between iris and capsule remnants in four eyes. The field of vision showed considerable improvement in the five eyes of the four patients who were tested preoperatively, and after operation only minor relative arcuate defects remained. In the other patient the postoperative field tests revealed minor arcuate defects in one eye and no abnormality in the other.

No patient has shown subsequent visual field deterioration or loss of control of intraocular pressure. None of the patients had optic nerve cupping of greater than 0.6 vertical cup to disc ratio

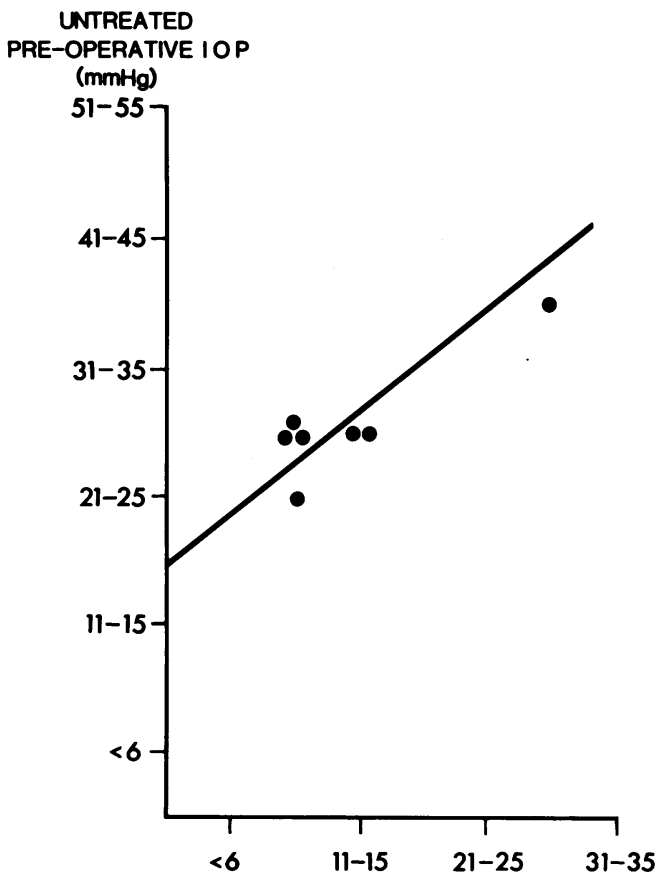

REDUCTION in IOP (mmHg) 3 MONTHS AFTER OPERATION

Fig. 2 Straight line relationship between untreated preoperative intraocular pressure and the reduction in pressure achieved by a first trabeculectomy in primary open angle glaucoma, redrawn from Jay and Murray. ${ }^{15}$ The results of the combined operations (black circles) show a similar effect on intraocular pressure: when last examined, which confirms the relatively early stage of optic nerve disturbance in all the cases described.

\section{Discussion}

Although the number of cases is small the results suggest that the effect of this type of combined operation is to achieve the visual benefits of cataract extraction with intraocular lens implantation as well as the intraocular pressure control expected after trabeculectomy. Combining the two procedures does not seem to introduce new complications. The visual results are similar to those in the author's unpublished Pearce posterior chamber lens series, and Fig. 2 confirms that the reduction of intraocular pressure is identical to that following a first trabeculectomy for

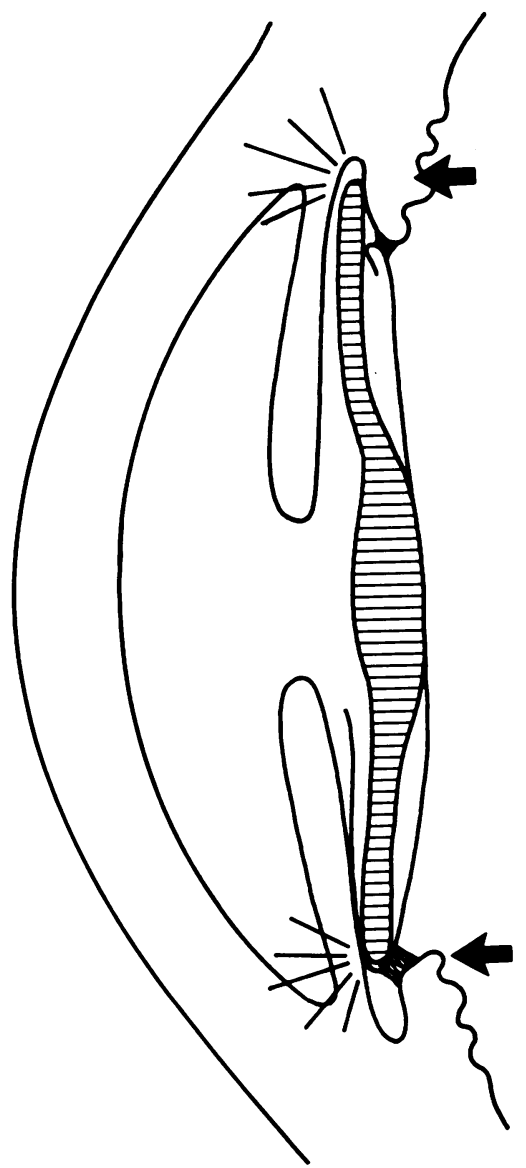

Fig. 3 Cross-sectional diagram of a Pearce tripod posterior chamber lens in place. The position of the rigid legs of this lens (arrowed) will prevent forward displacement and shallowing of the anterior chamber even in the event of excessive drainage of aqueous. 
primary open angle glaucoma where the intraocular pressure is lowered to the physiological range irrespective of the preoperative pressure. ${ }^{15}$ The observed visual field improvement was probably a result of improved retinal illumination following removal of the cataractous lens.

After trabeculectomy the risk of shallow or flat anterior chamber in the presence of an intraocular lens might be hazardous, but the Pearce tripod lens is a single plane lens with no component in front of the pupil, and its rigid legs act as radial struts to stop the implant moving forwards even with excessive drainage of aqueous. This implant will therefore prevent the anterior chamber becoming shallow (Fig. 3), a feature which is also useful when intraocular lens insertion is combined with penetrating keratoplasty. It is not known whether posterior chamber lenses with flexible loops will prove stiff enough to prevent loss of the anterior chamber in this manner, and for various reasons iris plane lenses and rigid angle supported lenses are best avoided in glaucomatous eyes. ${ }^{16}$

Many glaucoma patients may have used miotic drops for years before surgery, and adequate dilatation of the pupil for extracapsular cataract may be impossible. In these cases the pupil may be enlarged surgically during the operation by a radial iridotomy from iris root to pupil, and after insertion of the implant the pupil is restored by suturing the iris.

It seems that, where a surgeon is experienced in this technique of cataract surgery, it is now possible to offer patients the benefit of combining intraocular lens implantation with glaucoma surgery. It is, however, still necessary to select patients with caution, as experience is limited to seven eyes which were eventually found to have good residual optic nerve function.

\section{References}

1 Galin MA, Hung PT, Obstbaum SA. Cataract extraction in glaucoma. Am J Ophthalmol 1979; 87: 124-9.

2 Collado Hornillos JA, López I, Izaquime J, Velarde R, et al. Results of trabeculectomy combined with cataract extraction. Arch Soc Esp Oftalmol 1980; 40: 1271-9.

3 Edwards RS. Trabeculectomy combined with cataract extraction: a follow-up study. Br J Ophthalmol 1980; 64: 720-4.

4 Grizalbo MP, Gomez ML, López A, Ferrer C, Honrubia FM. Arch Soc Esp Oftalmol 1980; 40: 469-75.

5 Luntz MH, Berlin MS. Combined trabeculectomy and cataract extraction. Trans Ophthalmol Soc UK 1980; 100: 533-41.

6 Scuderi G, Balestrazzi E, Scorcia G. Combined operations for cataract and glaucoma. Clin Oculist Patol Oculare 1980; 1: 5-16.

7 Wechsler A, Robinson LP. Simultaneous surgical management of cataract and glaucoma. Aust J Ophthalmol 1980; 8: 151-60.

8 McPherson S. Combined trabeculectomy and cataract extraction. Int Ophthalmol Clin 1981; 21: 87-92.

9 Klemetti A, Kalina T. Combined trabeculectomy and cataract operation. A follow-up study. Acta Ophthalmol (Kbh) 1982; 60: 259-66.

10 Stiegler G. Combined trabeculectomy: intracapsular cataract surgery with lens implantation. Klin Monatsbl Augenheilkd 1981; 179: 456-60.

11 Percival P. Policy on cataract with glaucoma. Contact Intraocul Lens Med J 1981; 7: 71-2.

12 De Heer LJ. Glaucoma as a problem in intraocular lens implantation. Doc Ophthalmol 1980; 49: 337-46.

13 Watson PG. Trabeculectomy. A modified ab-externo technique. Ann Ophthalmol 1970; 2: 199-205.

14 Pearce JL. Experience with 194 posterior chamber lenses in 20 months. Trans Ophthalmol Soc UK 1977; 97: 258-64.

15 Jay JL, Murray SB. Characteristics of reduction of intraocular pressure after trabeculectomy. Br J Ophthalmol 1980; 64: 432-5.

16 Layden WE. Pseudophakia and glaucoma. Ophthalmology (Rochester) 1982; 89: 875-9. 